\title{
Radio Channel Model for Agricultural Wireless Sensor Networks
}

\author{
Guang-You Yang \\ Institute of Agricultural Machinery, Hubei University of \\ Technology \\ Wuhan, P.R. China \\ pekka@126.com \\ Hao Chen \\ Institute of Agricultural Machinery, Hubei University of \\ Technology \\ Wuhan, P.R. China \\ 535193320@qq.com
}

\author{
Xiong Gan \\ School of Mechanical and Electronic Engineering, Wuhan \\ University of Technology \\ Wuhan, P.R. China \\ ganxiong001@126.com
}

\begin{abstract}
In order to study the influence of the agricultural greenhouse environment on the channel of wireless sensor networks (WSNs) and to establish the WSNs channel model suitable for the agricultural greenhouse, this paper measures the path loss characteristics of the $2.4 \mathrm{GHz}$ radio frequency in the agricultural greenhouse. The original measurement data cannot be directly used for establishing the WSNs channel path loss model due to its big error. So the kalman filter is introduced to reduce the noise of the original measurement data, and then the accurate model parameters are obtained by linearity regression. The results indicate that the log distance path loss model can accurately characterize the path loss characteristics of radio frequency in the agricultural greenhouse environment, and the propagation characteristics of the WSNs channel model can provide theoretical basis for setting up the WSNs monitoring system platform of the agricultural greenhouse.
\end{abstract}

Keywords-Agricultural greenhouse, Wireless Sensor Networks, Channel model, Kalman filter, Path loss model

\section{INTRODUCTION}

Wireless sensor networks (WSNs), as a new type of integrated information network system, has been gradually adapted in the agricultural sector due to its selforganization, low power consumption, no wiring and strong intelligence [1]. Currently, the WSNs applied research in the field of agriculture focuses on the monitoring of the field crop growth parameters or agricultural greenhouse environmental factors such as temperature, humidity, rainfall, sunlight and nutrient contents of leaf area index, and the combination of the relevant key technologies such as node deployment, routing protocol, energy harvesting, energy conservation, data fusion and services with practical application is the current hotspot [2]. For example, California camalie vineyard deploys 20 Crossbow's Mica2dot nodes in the area of $1.78 \mathrm{hm}^{2}$, setting up the soil temperature and humidity monitoring network [3]. In 2002, Intel Corporation established the first wireless sensor vineyard as a pioneer in Oregon. The sensor nodes are distributed at each corner of the vineyard, detecting the soil temperature, soil humidity or the number of the harmful substances in the region every minute [4]. Some domestic research institutes and universities have launched related research. South China Agricultural University has achieved certain results in the aspects of the deployment and network scheme of wireless sensor networks [5]. Jiangsu University also applied wireless sensor networks technology in its water-saving irrigation and agricultural meteorological monitoring projects and has obtained good feedbacks $[6,7]$.

Although in recent years there have been some research and application achievements in WSNs, but the promotion and application of WSNs in monitoring agriculture is not extensive, mainly due to the large spatial and temporal variability of agricultural environment. The environmental factors severely impact the stability and reliability of the wireless signal propagation. The propagation of the wireless signal from a transmitting node to a receiving node is filled with volatility and attenuation effect, which is caused by the loss path of the wireless signal transmission. At the same time, the wireless signal transmission is also jointly affected by the topography, vegetation density, plant height, temperature, humidity, height of the transmitting and receiving nodes and other complex factors. The fading research of the wireless signal in the WSNs channel can provide the theoretical basis and practical guidance to the plan and deployment of WSNs in agricultural greenhouses. In this paper, an agricultural greenhouse from the academy of agricultural sciences is selected as the trial venue to conduct the WSNs channel 
communication test with $2.4 \mathrm{GHz}$ frequency. Studying the characteristics of wireless signal decline and fluctuation law of inter-node link quality in agricultural greenhouses, establishing the relevant mathematical models can provide the theoretical basis for the subsequent application.

\section{MATERIALS AND METHOD}

\section{A. Test Environment}

In this paper, an agricultural greenhouse of an academy of agricultural sciences is selected as the test environment, which is shown in fig. 1.

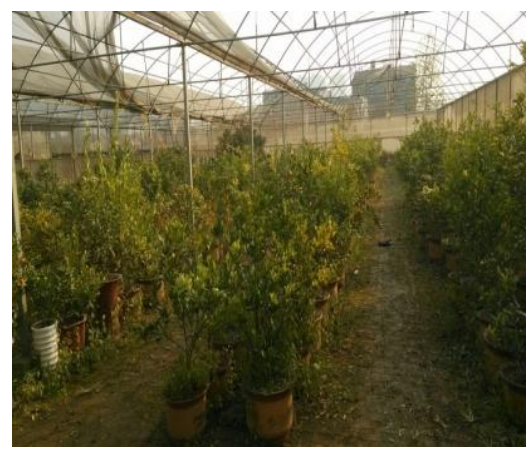

Figure 1. Test site

The agricultural greenhouse is 40 meters long, 24 meters wide and fully enclosed, leaving only one door for access. The terrain of the agricultural greenhouse is flat and the plants in it grow in the pots, which are arranged in five straight columns. The 12 transmitting nodes are 0.5 meters off the ground and the receiving node is placed on the sill of the duty room, 1 meter off the ground. The test site and nodes deployment are shown in fig. 2.

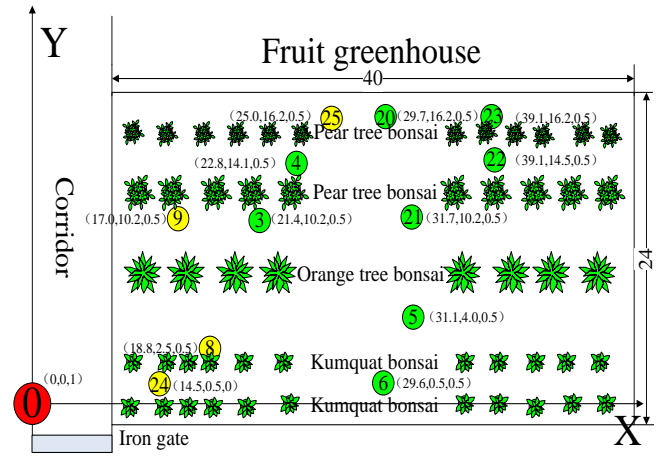

Figure 2. Node deployment diagram

\section{B. Test Equipment}

Experimental signal transmitting and receiving nodes adopt TI CC2530 RF chip with integrated 51 microcontroller core as the ZigBee SOC solution, the operating frequency is $2.4 \mathrm{GHz}$. The transmission power of the transmitting nodes is $-17.4 \mathrm{dBm}$ and the receiving node is connected to the computer through the USB port. The PC software for laboratory is a self-developed comprehensive platform by using $\mathrm{VC}++6.0$, which can monitor the real-time network topology structure of WSNs and receive the datum from the agricultural greenhouse collected by the transmitting nodes and store them in the database.

\section{Model Selection and Data Filtration}

The Affected by factors such as terrain, vegetation and etc, path loss, shadow fading and multipath effect occur to lead to the decline of the signal in the process of the radio signal propagation. The size of the power represents the strength of the radio frequency signal. The expression between the transmitting power and the receiving power is

$$
\mathrm{P}_{\mathrm{R}}=\mathrm{P}_{\mathrm{T}} / \mathrm{d}^{\mathrm{n}}
$$

Where, $P_{R}$ is the receiving power of the RF signal with its unit of $\mathrm{mW}, \mathrm{P}_{\mathrm{T}}$ is the transmitting power of the $\mathrm{RF}$ signal with its unit of $\mathrm{mW}, \mathrm{d}$ is the distance between the transceiver element with its unit of $m$ and $n$ is the path loss factor, value of which depends on the RF signal propagation environment. The expression (1) is converted to be

$$
P_{R}(d B m)=A-10 n l g d .
$$
is

So, it is concluded that the expression of the path loss

$$
P_{L}(d)=P_{L}\left(d_{0}\right)+10 n \log _{10}\left(d / d_{0}\right)
$$

Where, $P_{L}(d)$ is the power loss of the wireless signal from the transmitting node to the receiving node with the distance $d$, the unit of which is $d B$ and $P_{L}\left(d_{0}\right)$ is the power loss of the near-earth reference point with its unit of $\mathrm{dB}$.

The above formula (3) is the log distance path loss model is widely used in the actual path loss estimation of the wireless channel and it indicates the size of the average received signal strength with the increase of distance between sending and receiving ends exponential attenuation, namely the linear attenuation relationship between the path loss and the logarithm distance of the transceiver end [8].Due to personnel were ambulating frequently and plants overlapping, the receiving node is faced with problems such as weak wireless signal and missing data. The original RSSI value and LQI value fluctuating on a wide range, and so the kalman filter is introduced to reduce noise for the original RSSI value and LQI value. In order to construct the kalman filter model, the discrete-time linear dynamic system is firstly introduced to use the following linear differential equations to represent the control process.

$$
\begin{gathered}
x_{k}=A x_{k-1}+B u_{k}+w_{k} . \\
z_{k}=H x_{k}+v_{k} .
\end{gathered}
$$


The formula (4) called the kalman filter state equation, describing the process of the system state changing over time, is a dynamic model of the system and the formula (5) called the kalman filter measurement equation describes the relationship between the measured value of the current system and the system state [9]. The working process of the kalman filter is shown in fig. 3 .

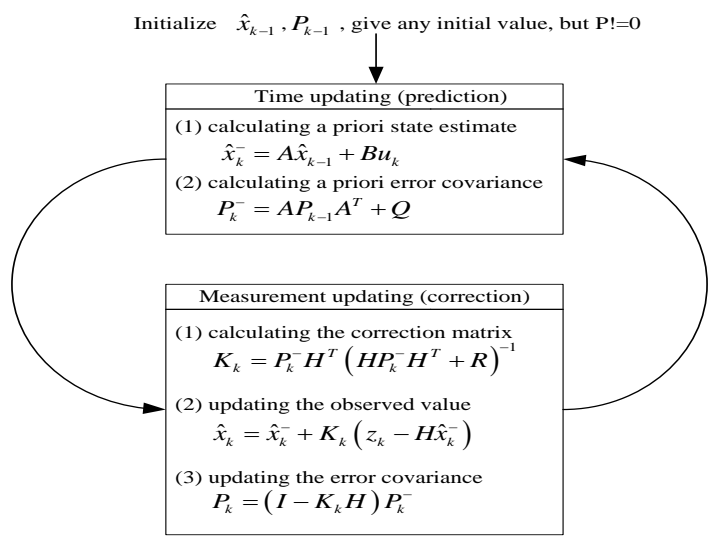

Figure 3. Working Process of Kalman Filter

After the noise reduction processing, the fluctuation range of the original measured RSSI values of the continuous time intervals in the same spatial position is greatly reduced, which reflects the received signal strength of the corresponding positions. The comparison of the original measured RSSI values with the RSSI values after the noise reduction processing is shown in fig. 4 and fig. 5 .

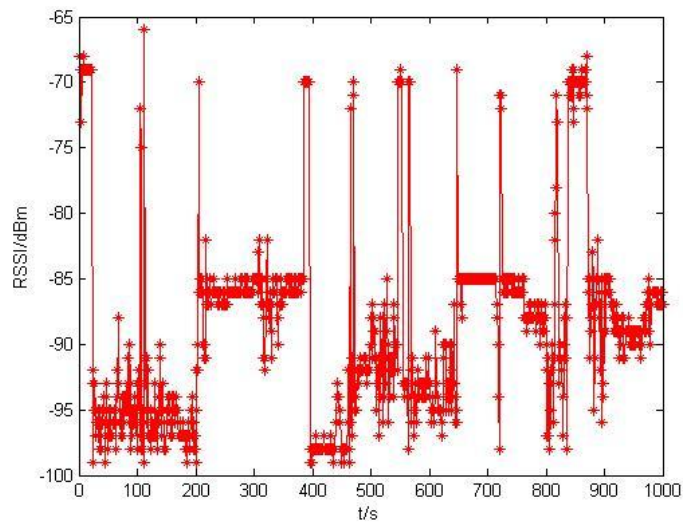

Figure 4. Original Measured RSSI Value

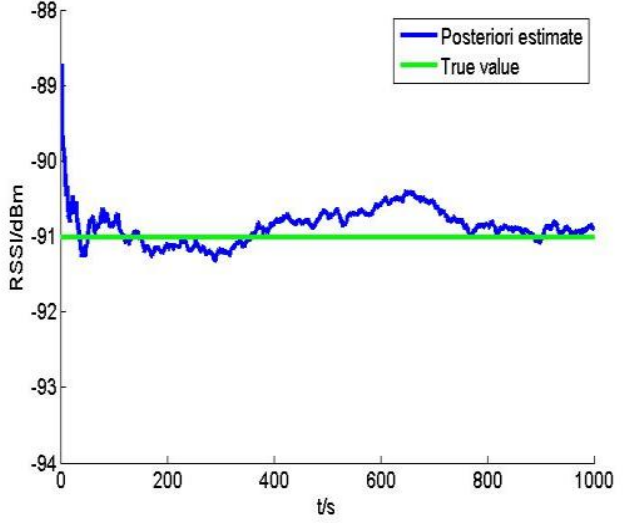

Figure 5. RSSI Value after Kalman Filter

After the kalman filter, the error in the original measured data brought by the plants covering and personnel ambulating is eliminated, which makes the data after the noise reduction processing closer to the real data, which lays foundation for the next step to establish the path loss model of the agricultural greenhouse.

\section{RESUlTS AND DISCUSSION}

\section{A. Pre-estimating the Path Loss Factor}

This test is mainly to establish the distance path loss model of the wireless signal propagation in the agricultural greenhouse, namely measure the relational expression of the path loss and the distance between the transceiver nodes. The nodes used in the experiment are all full function devices (FFD), among which one Coordinator node, four Router nodes and eight End Device nodes, a total of 13 nodes constitute a tree network and the network topology is shown in fig. 6 .

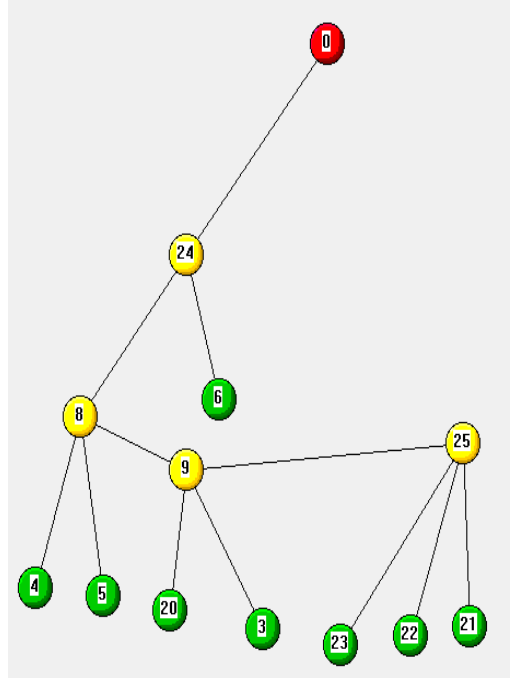

Figure 6. Network topology diagram

Each node except the Coordinator node continuously collects 1000 groups of data, and then kalman filter is used in reducing the noise of the original measured RSSI values 
in the 1000 groups of data and the average value of the 1000 groups of the RSSI values after the noise reduction processing is regarded as the real RSSI value of the corresponding node to exclude the impact of the smallscale fading as far as possible, so that the measured values reflect the large-scale path loss characteristics in the average sense as much as possible[10]. The path loss $P_{L}\left(d_{0}\right)$ of the near-earth reference point $\mathrm{d}_{0}=1 \mathrm{~m}$ is measured to be $53 \mathrm{~dB}$, and the value of the path loss factor $\mathrm{n}$ is estimated to be 1.5 according to fig. 7 .

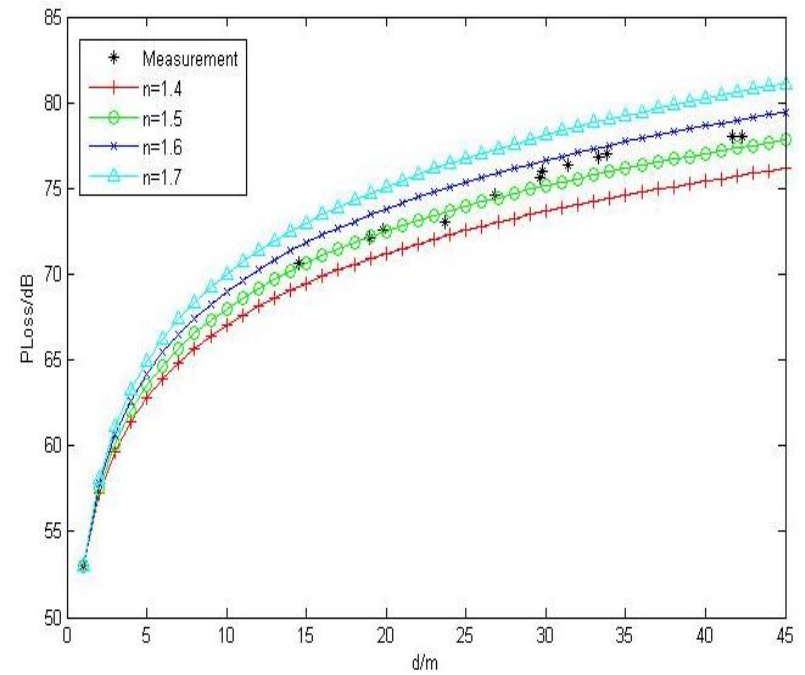

Figure 7. Path Loss Factor Estimate Diagram

Therefore, the log distance path loss model formula is estimated to be
Using Matlab to compare the formula (6) with the measured values, it is obtained that the correlation coefficient is 0.9976 , the determination coefficient is 0.9349 and the root mean square error is 0.6426 . The above data show that the estimated model can accurately reflect the path loss characteristics of the RF signal in the agricultural greenhouse.

\section{B. Verifying the Estimation Model}

Using Matlab toolbox CFtool to process the data of 12 test nodes, the fitting result of the log distance path loss model of the RF signal propagation in the agricultural greenhouse is shown in fig.8.

As shown in Fig. 8, the fitting curve formula is

$$
P_{L}(d)=52.74+15.51 * \log _{10}(d) .
$$

Where, the path loss factor $\mathrm{n}$ is 1.551 , the path loss of the near-earth reference point is $52.74 \mathrm{~dB}$, the determination coefficient is 0.9952 and the root mean square error is 0.4762 . The above data verify the $\log$ distance path loss model is highly suitable for the propagation characteristics of the RF signal in the agricultural greenhouse.

Comparing the formula (6) with the formula (7), it can be concluded that the path loss factor $n$ of the agricultural greenhouse environment is 1.55 , the path loss of the nearearth reference point of the agricultural greenhouse environment is $52.74 \mathrm{~dB}$ and the final log distance path loss model formula is

$$
P_{L}(d)=52.74+10 * 1.55 * \log _{10}(d) \text {. }
$$

$$
P_{L}(d)=53+15 * \log _{10}(d) \text {. }
$$

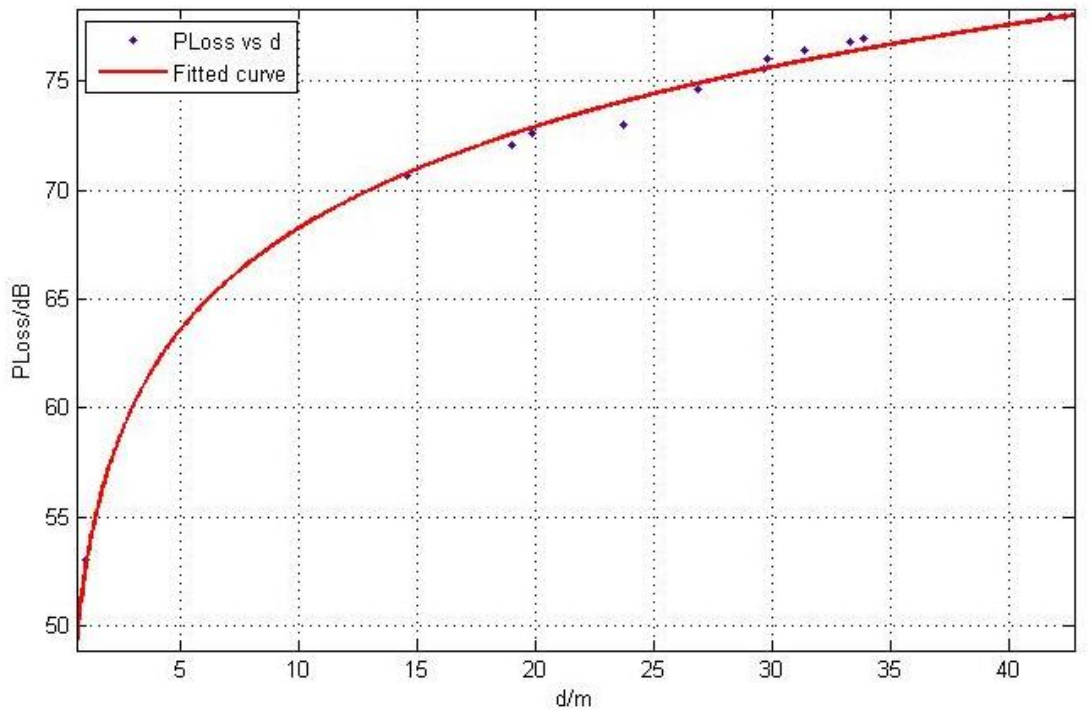

Figure 8. Fitting Curve of Log Distance Path Loss Model 
The matrix tool Matlab is used to figure out that the correlation coefficient between the formula (8) and the measurements is 0.9976 , the determination coefficient is 0.9934 and the root mean square error is 0.4353 . The final result shows that although the agricultural greenhouse environment is complex and the factors which influence the spread of the RF signal are diverse, the log distance path loss model is still applicable to the agricultural greenhouse environment.

\section{CONCLUSION}

Aiming at the need of the WSNs channel test in the agricultural greenhouse, this paper studies the propagation characteristics of the $2.4 \mathrm{GHz}$ wireless radio frequency signal in the agricultural greenhouse. Using the kalman filter to process the original measurements, the log distance path loss model suitable for the agricultural greenhouse environment was obtained. Subsequently the path loss index 1.55 of the agricultural greenhouse environment was obtained, but the model which this paper achieved is only the large-scale fading model, so it is still necessary to measure the small-scale fading model of the agricultural greenhouse environment to lay the theoretical basis for building the efficient and low-redundancy WSNs monitoring system oriented to the agricultural greenhouse.

\section{REFERENCES}

[1] H. Wang., L. L. Wang and X. S. Kong.: Path Loss Modeling for Near-ground Wireless Channel in Grassland Environment. Optics and Precision Engineering. 20(6), 1406-1413(2012)
[2] X. J. Yue., Y. F. Wang and T. S. Hong.: WSNs Layout Experiment Based on Radio Frequency Propagation Tests in Citrus Orchard. Transactions of the Chinese Society for Agricultural Machinery. 44(5), 213-218(2013)

[3] Y. H. Cai., G. Liu and L. Li.: Design and Test of Nodes for Farmland Data Acquisition Based on Wireless Sensor Networks. Transactions of the CSAE. 25(4) , 176-178(2009)

[4] X. J. Qiao., X. Zhang and C. Wang.: Application of The Wireless Sensor Networks in Agriculture. Transactions of the CSAE. 21(S), 232-234(2005)

[5] T. Wen., T. S. Hong and Z. Li.: Test of Wireless Sensor Networks Radio Frequency Signal Propagation Based on Different Node Deployments in Citrus Orchards. Transactions of the CSAE. 26(6), 211-215(2010)

[6] Y. B. Feng., R. B. Zhang and G. D. Gu.: Application of Wireless Sensor Networks in Water-Saving Irrigation. China Rural Water and Hydropower. (2) , 24-26.(2007)

[7] Y. J. Cao., X. Z. Wang and J. Q. Yang.: Design of Farmland Weather Monitoring System Based on WSNs. Journal of Agricultural Mechanization Research. (12),163-165(2008)

[8] W. Q. Tian., C. Zhang and J. F. Luo.: Quadratic Path Loss Model Based on Typical Urban Road Environment. Computer Engineering. 39(10) , 76-80(2013)

[9] X. Xie., J. M. Guo and W. T. Cao.: Research on Continuous Positioning with Insufficient Visiable Satellites Based on Kalman Filter Algorithm. Geospatial Information. 11(1) , 11-13(2013)

[10] C. Zhang., J. F. Luo and W. Q. Tian.: Measurement and Analysis of Wireless Sensor Networks Channel in Urban Environment. Computer Engineering. 39(5) , 28-33(2013) 